\title{
Evomimetics: the biomimetic design thinking 2.0
}

\section{Adriaens}

D. Adriaens, "Evomimetics: the biomimetic design thinking 2.0," Proc. SPIE 10965, Bioinspiration, Biomimetics, and Bioreplication IX, 1096509 (13 March 2019); doi: $10.1117 / 12.2514049$

Event: SPIE Smart Structures + Nondestructive Evaluation, 2019, Denver, Colorado, United States 


\title{
Evomimetics: the biomimetic design thinking 2.0
}

\author{
D. Adriaens ${ }^{\mathrm{a}}$ \\ ${ }^{a}$ Ghent University, Dept. of Biology, Evolutionary Morphology of Vertebrates, K.L. \\ Ledeganckstraat 35, B-9000 Gent, Belgium
}

\begin{abstract}
The consensus is that nature is a tremendous source of ideas for innovative designs that can meet various specific functional needs, relevant to society. Designs rely on structural, constructional, process-based and behavioral traits that all result from a natural trial-and-error cycle: evolution. Being one of the pillars of biomimicry, through billion years of evolution, nature has experimented and found what works and lasts, and what does not. Evidently, this has attracted scientists, especially engineers, trying to understand working natural designs, and translate them into applicable, working synthetic designs. The 'Biomimetic Design Method' forms the underlying conceptual framework to analytically decode biologically functions and designs. However, even though the evolutionary process is considered key to all this, it is generally overlooked in this conceptual thinking. The general assumption is that particular functions in organisms result from a natural selection process that optimized the underlying design for a particular function, thereby overlooking that an organism actually represents the possibly best compromise between all its functions needed to survive, to reproduce and to produce fit offspring. Many evolutionary processes thus yield suboptimal design components that, when put together, provide an optimized organismal design that manages to perform as good as needed, within a given environment. Such evolutionary limitations thus create possible pitfalls for bio-inspired design thinking. But, when considering them as a structural part of the design thinking process ('evomimetics'), they actually create opportunities for an improved translation of biology into optimally functioning designs. Using specific examples from evolutionary biology, these processes are explained, and recommendations are formulated.
\end{abstract}

Keywords: Biomimicry, biomimetics, design, evolution, conceptual, constraints, optimization, adaptation

\section{INTRODUCTION}

Nature has been a source of inspiration for naturalists, artists and also engineers, acting as a true idea generator [1]. A three-fold role has been awarded to nature within this context: it can act as a model (for imitating natural designs and processes), as a measure (assuming that nature's results are a reference to what works and lasts), and as a mentor (for valuing nature in its widest possible form) [2]. No matter how or for what purpose, the evolutionary history and mechanisms resulting in natural systems and processes, as we know them, are considered key when trying to use nature as this idea generator. The range of idea's has proven to be limitless: from Greek myths to whale inspired mega planes [3]; from Egyptian prosthetic toes [4] to a powered human exoskeleton [5]; from bird inspired, flappingwing ornithopters to miniature drones [6]; from the level of nanoparticles [7,8] through traffic optimization strategies $[9,10]$ to sustainable waste recycling strategies [11, 12]; from energy production [13] to energy harvesting [14]; or from gecko skin inspired self-cleaning coatings [15] to fish-based flexible armor [16]. This bio-inspired interest is mainly in natural shapes, structures, patterns, materials and functions [17-19]. Apart from the naturerelated content, the Bio-Inspired Design (BID) thinking also shares other aspects with biology, even at the level of conceptual and philosophical approaches to understand the intriguing relationships between form and function: form creates function or vice versa $[20,21]$.

Different strategies exist on how and to what degree concepts from nature are used in applications. They are frequently categorized based on some key principles, or rather nuances $[1,14,22]$. Biomimicry, sometimes considered a synonym for 'biomimetism', refers to the "philosophy and interdisciplinary design approaches taking nature as a model to meet the challenge of sustainable development (social, environmental and economic)" [22-24]. Other strategies, sometimes labeled as 'engineered biomimicry' [14], are bio-replication ("direct replication of a structure found in living organisms, and thereby copy one or more functionalities"), biomimetics ("reproduction of the physical/chemical mechanisms underlying a specific functionality of a plant or animal") and bio-inspiration

Bioinspiration, Biomimetics, and Bioreplication IX, edited by Raúl J. Martín-Palma, Mato Knez, Akhlesh Lakhtakia, Proc. of SPIE Vol. 10965, 1096509 · @ 2019 SPIE · CCC code: 0277-786X/19/\$18 · doi: 10.1117/12.2514049 
("reproduction of the outcome of a certain functionality of a plant or an animal without reproducing either the physical/chemical mechanisms or the biological structure responsible for the outcome."). Even the concept 'biologize' is used, referring to existing human problems being rephrased in such a way, that an answer can be found in biology [25]. However, as many as there are terminologies and definitions, as much is the demarcation of each strategy quite fuzzy. For example, Tan, et al. [26] mention that biomimetics and biomimicry are sometimes considered as synonyms, and even a synonym with bionics. For pragmatic purposes, these approaches will further be referred to as 'Bio-Inspired Design (BID) thinking'.

Irrespective of such terminology overlap, what is the core of all of them is that they all rely in some way on the assumption that natural designs result from an evolutionary process that strives for optimization. This makes sense considering how the general idea of Darwin is commonly interpreted: evolution striving towards organisms iteratively becoming better adapted to their environment, resulting in an increased fitness [27]. However, in reality, this is not how evolution works at a mechanistic level, as well as there are many limitations on what is biologically possible for organisms to become modified. The focus of this paper is exactly on the pitfalls of this general assumption, by showing that this assumption is prone to generate false ideals towards optimization of BID. Several of these pitfalls are illustrated, as well as an optimized approach is suggested (coined 'evomimetics'), that could help engineers, not only in overcoming these pitfalls, but by doing so, improving the design process leading to better (and truly) optimized BIDs.

\section{BIOMIMETIC CHALLENGES}

The core strategy of any of the above-mentioned BID thinking processes involves the identification of biological potentials and somehow linking them to engineering problems. Along a series of steps from biology to engineering, involving identification, analysis, abstracting, implementation and testing, practitioners of BID are confronted with two major challenges: (1) "engineers and biologists speak different languages" [28], yet biomimetics is about biologists and engineers translating biological solutions to solve problems through BIDs; and (2) the optimization potential of biological evolutionary processes may be overestimated.

\subsection{Lost in translation}

BID thinking implies a problem-solving approach for technological purposes, by looking at how organisms' problems have been solved through evolution. Even though most agree that an organism can be seen as a dynamic outcome of a process of evolutionary trial-and-error in response to environmental challenges, identifying and defining exactly what these problems are becomes more difficult [29]. The consensus is that for research on BIDs, a proper understanding of the underlying biological structure and function is pivotal. However, this understanding is generally considered to be sufficient when the structure-function relationships in biological systems are well understood (for behavioral functions, also organism interactions are considered) [26, 30]. By understanding natural structures, patterns and functions, it is seemed adequate to translate these into analogies that can be understood by engineers. This remains a challenge, considering that functionalities in engineering are not necessarily analogous in complexity to how they are manifested in natural systems. A natural function is frequently considered to be hierarchical and dynamical, in contrast to engineering functions. It is being recognized that natural functionality relies on "information embedded at various hierarchical levels" [31], but it remains unclear on what this information and these levels are. Yet, the statement is true, when looking closer into the underlying evolutionary processes that cause natural functions to emerge. The three most important challenges a designer is confronted with, when browsing the literature on biological entities (structures, functions, mechanisms, physical properties and ruling environmental conditions) are findability, recognizability and understandability [31]. These challenges should actually be seen at two levels: at the descriptive context of what the biological structures and function are, but also with respect to what the evolutionary track is that led to these structures and functions. Kruiper, et al. [31] focused on the former, this paper here focuses especially on the latter.

The translation challenge in BID research is how to properly grasp biological complexity and translate that towards tangible technological functionality, and thus is not to be seen as a 'direct carry-over from biology' to technology [32]. For dealing with biological complexity, researchers exploring biomimetic potentials "need to take much greater advantage of the knowledge and expertise of biologists - be they ecologists, microbiologists or specialists in 
evolution, organisms, cells or molecules" [33]. Most biomimetic papers focus on a single species or refer to 'a cell' in a too generalized manner. In a similar manner, the process of evolution itself is lacking, where "biologists can match a problem that a researcher maybe is trying to tackle to a specific species, environment or suite of evolutionary conditions". Specific subdisciplines in biology, such as evolutionary biology and functional morphology could be particularly relevant. Already understanding how individual species solved biological problems can be instrumental for researchers in BID, whereas, working with evolutionary biologists to study entire evolutionary lineages of species can provide a more generic understanding of how form and function dynamically relates to a changing environment. Speck and Speck [32] rightfully claim that in a bottom-up approach, the first step requires a fundamental approach by studying the 'mechanics and functional morphology of a biological system'.

A problem with the BID translation may then especially arise when the same words have different connotations in biology versus engineering sciences. Not only does this compromise the actual translation, but it also creates incompatibilities when automated search engines try to identify and link biological contexts to engineering contexts. Even the basic components of a design, i.e. 'structure' and 'function', are interpreted somehow differently. A 'structure' in engineering actually refers to a an 'element of a Bauplan' in organismal biology (where the 'Bauplan' reflects the combination of all such elements into a living organism) [34]. In the same context, a 'structure' in biology then specifically refers to only one sublevel of the element's morphology, i.e. the "topographic set of all material cellular, intra-cellular and inter-cellular parts which fill up the internal space of the element". The 'morphology' of an element is then defined by this structure, as well as by its size, shape and position (within the organism). Elements are then assembled in different 'apparatuses', which through a specific 'mechanism' (defined by the interaction between the elements into an apparatus) perform one or more specific 'functions'.

'Function' is considered to be the key concept in biomimetics, the level at which translation from biology to engineering is feasible [31]. In engineering, a 'function' has been defined as "an operation performed on a flow of material, signal, or energy" [35]. More specifically for design and product development, functions are considered an important abstraction level, seen as the specific tasks a design or product has to perform [36]. Here, functions also involve the process efficiency, from the design labor up to bringing the designs to market. Functions are also considered as units for problem decomposition, partially defined by what a particular customer needs [37]. Several functions defined for engineering design [37], actually match well with those identified in biology. However, in biology, a nuance can be made between 'function' and 'biological role', where a function takes in the biological role when it contributes to the maintenance of the complete organism in a spatio-temporal context. Take the jaw system in rodents: the lower jaw comprises a single element (a dentary bone, equipped with large teeth) that interacts with other elements to form the jaw apparatus: it forms a mobile joint with the skull and is connected to it through several muscles. One specific 'function' of this jaw apparatus is to move the lower jaw in a sufficiently powerful way so that teeth can chew food or other objects, where 'performance' is then a measure of how well the function is performed. Frequently, a single apparatus performs multiple functions. In some rodents, such as subterranean mole-rats, the biological role of the jaw apparatus is then to feed, to fight and to dig protective tunnels [38, 39].

It becomes even more problematic when trying to define the nature of links that may exist between structures and functions, i.e. "trade-off relations, causality, correlations, associations, separations, processes and identities" [31, 40]. Just as in engineering sciences, it is crucial to distinguish causalities from non-causal correlations in biology. However, considering the complex hierarchy that may underly correlated interactions in natural systems, this is a continuous challenge for evolutionary biologists. Identifying the cause and consequence is even close to impossible in many cases, as the details of the evolutionary polarity (at population levels) are generally unknown. This debate has ruled biology for centuries, including the one on whether form causes function to change, or whether it is function that causes form to change. Less difficult to grasp, but yet highly relevant for evolutionary biology, is the concept of 'trade-offs'. Also here, the interpretation of a trade-off in biology may entail a broader spectrum than what is the case in engineering, again because of the multilayered evolutionary context. In engineering sciences, the concept of a Pareto set of combinations of two opposing functions reflects the set of desirable combinations of tradeoffs between these two functions [40]. Such trade-offs are frequently considered only at a single abstraction level within a technical context. For example, the best combination of the purchase price and fuel consumption of a car does not depend on other factors, such as historic background, whether the car designer was constrained in the design criteria because of previous car types, or whether the production of components was limited. That is where the analogy with biological trade-offs may fundamentally differ: the most optimized biological system does depend on 
evolutionary history, it does depend on ancestor-derived constraints and it does depend on flexibility in the genotypephenotype map as it becomes unrolled during development. As stated by Vincent [40], the rather simple dichotomy that underlies pragmatic approaches, such as in a Pareto analysis, is far from similar to the complexity of biological, evolutionary hierarchy (with its 15 to 20 levels of hierarchy), which is characterized by unlimited number and types of trade-offs. In addition, trade-offs are only recognized as such when there is variation along the trade-off trajectory (so-called Pareto front). This implies that 'fixed trade-offs' (that show no variation in the trait combination) may not be identified as a true trade-off [41]. This makes sense from a practical-technical point of view, but does not from an evolutionary point of view. For example, the almost fixed condition of seven cervical vertebrae in mammals (very few exceptions exist) would thus not be considered as resulting from a trade-off. Yet, it is known that an interaction between the genetic programming of additional neck segments and an uncontrolled tissue proliferations during early embryogenesis results only in a viable trade-off if the number of neck segments is seven [42].

This complexity of natural systems also makes it difficult for the two core aims of BID thinking: identifying a biological solution, and identifying the biological problem for which this solution emerged during evolution [31]. This implies that the problem needs to be properly identified first; only then the actual solution can be established. Identifying the true nature of a biological problem may seem trivial, but generally it is not because an integrated organism is built by several hierarchical levels of structures and functions, and their interactions and trade-offs. As an organism has to perform many functions 'in harmony', it becomes a challenge to disentangle them into specific solutions for specific problems. If this could be accomplished, the next question then is whether it can be verified if the solution of interest is optimized for the specific problem, or rather the best evolutionary compromise that is 'just good enough'? Even if that could be achieved, it remains to be solved whether the 'just good enough' is because it is indeed good enough to solve the problem (no better is needed), or whether the suboptimal solution is the best, biologically feasible solution considering all the evolutionary constraints (better is not possible).

\subsection{What 'evolution' does ... and does not}

Natural selection, variation, speciation, adaptation, fitness, etc. ... all are part of a highly complex process defining organismal evolution. Although the overall process is well established among evolutionary biologists, they are still trying to unravel every detail at every level of every evolutionary process, which spans from gene to ecosystem. It is then also evident that when BID researchers want to rely on this evolutionary process, they have to rely on a certain abstraction of what that process actually entails. In several cases, however, this abstraction becomes too generalized, leading to misconceptions and wrong assumptions. This is especially so with respect to whether evolution (1) can rely on an unlimited flexibility to find the best solution, (2) strives towards optimized organismal designs (both in structure and function), and (3) revolves only about the environment dictating how organisms will become modified. Some quotes from literature illustrate this.

- "Over the 3.8 million years since life is estimated to have begun to appear on Earth, evolution has resolved many of nature's challenges leading to lasting solutions with maximal performance using minimal resources" [43]. This quote makes a lot of sense, such as that 'minimal resources' reflects the energy conservation strategy that favors fitness improvement. However, evolutionary change of functional performance is not necessarily, and even rarely, towards a maximum. Rather, evolution through variation and natural selection actually strives towards 'just enough', because of this energy conservation strategy.

- The statement that "nature is creating better the biological structure than the technology developed by humans" does not hold when looking at (just to give one example) the scale of mega-constructions realized by humans, compared to the size limits terrestrial organisms deal with because of limitations in their structural components (even though some dinosaurs were gigantic, they did not reach the dimensions of mega bridges or sky scrapers because of constructional constraints) [44].

- When referring to "all coupled biological factors and their causal relations must be taken into consideration when developing biologically inspired engineering designs" [26], these 'all coupled biological factors' are generally considered only at a single organismal level, and not on an evolutionary spatio-temporal level (from gene to ecosystem) [25].

- With respect to how evolutionary 'constraints' should be considered, Byrne, et al. [45] state that "adaptation and evolution allow organisms to exist within the constraints imposed on them by their respective environments", excluding constraints imposed by the organism itself. 
Yet, sometimes particular nuances are made that try to raise awareness in the BID community about these potential pitfalls. Cohen and Reich [1] rightfully mention that "nature design solutions are not always optimal, ideal, elegant or perfect". Using the swimbladder of fish as an example, Kruiper, et al. [31] state that "one has to consider that certain properties to the system's components may not be optimized for the function of interest or not contributing to this function at all". Vincent [40] then warns about the adaptive nature of BIDs not being as trivial as may at first seem, saying that "unless environmental and physiological selection pressures can be defined, the adaptations cannot be understood...".

Conceptual pitfalls and erroneous assumptions by simplicity, when it comes to understanding the nature of adaptive evolution, has also plagued biological researchers in the same way as it does BID researchers. Particularly relevant is the so-called 'Panglossian paradigm', or the misconception that any change in evolution must be considered as an adaptive response, frequently resulting into scientific storytelling of adaptive stories that lack any proper hypothesis testing [46]. This misconception is based on the "faith in the power of natural selection as an optimizing agent", where "trade-offs among competing selective demands exert the only brake upon perfection". Particularly relevant for the BID thinking is that "we all say that not everything is adaptive; yet, faced with an organism, we tend to break it into parts and tell adaptive stories as if trade-offs among competing, well designed parts, were the only constraint upon perfection for each trait". Even more, this adaptionist assumption fails to consider that structural and functional changes can also arise without a selection process towards improved performance, i.e. through neutral evolution as a non-adaptive, alternative scenario [47]. Some examples of what are generally considered as textbook examples of adaptive stories, turned out to be wrong after proper empirical testing. For example, the neck of giraffes may rather result from sexual selection (instead of selection to reach the highest leaves) [48] and oxpeckers are not simply mutualistic tick removers for large mammals [49].

\section{EVOLUTIONARY SUBOPTIMIZATION IN BID}

So how does evolution then work, if it should be selecting for the survival of the fittest but yet, does not necessarily come up with the most optimized solution for particular biological problems? The two main concepts in this context, that should clarify this, are 'survival of the fittest' and evolutionary limitations or 'constraints'.

\subsection{Survival of the fittest}

Only after Charles Darwin wrote his 'Origin of species', the concept of 'survival of the fittest' was coined, when the analogy was drawn between economics and his theory of evolution. This quickly led to a common misinterpretation as if evolution is the result of a selection process (natural selection) where only the individuals that are the fastest, the strongest, etc. are the ones fit enough to survive the harsh environment. However, this process is more complex, and less black and white than this.

\subsubsection{Selection}

Although it may seem straightforward that selection acts at the level of organisms, biologists agree that there is no unifying level at which variation is being filtered based on the response to particular environmental changes. First, 'natural selection' s.l. (as the opposite for 'artificial selection') comprises natural selection s.s. (environmentally driven), sexual selection (mating success driven) and kin selection (social context and genetic affinity driven). Natural selection may also act from a wide range of levels, i.e. from genes (e.g. selfish genes) [50], apparatuses (e.g. feeding apparatus), individual organisms, sex (sexual selection), relatives (kin selection), breeding pairs (being the smallest sexually reproducing unit), colonies (e.g. social insects), populations (as they carry the gene pool from which adaptive variation can originate) to species [51]. It is important to emphasize that natural selection does not act directly on the genotype or the phenotype itself, but on the performance of the phenotype within its environmental context. Also, depending on the ecological setting in which natural selection takes place, only through directional selection will functional traits become more and more performant, potentially leading to the fastest, the strongest, etc. Yet, nature may also favor the average performance through stabilizing selection (e.g. a too high or too low birth weight in humans comes with negative consequences) or may even allow a more optimal use of natural resources by selecting for different phenotypes ('morphotypes') through disruptive selection (e.g. well-known examples in Darwin finches) [52]. 


\subsubsection{Adaptation versus fitness}

Irrespective of the selection level, it can be summarized that natural selection controls how organisms perform within their environment in such a way that reproductive success is optimized. This reproductive success, i.e. the number of viable offspring, is what 'fitness' actual means in biology. Here it is fair to talk about 'optimized', being 'finding the best solution, considering the available resources, to produce as much offspring with an as high as feasible chance of surviving'. That does not mean offspring is maximized, as this may even result in a lower fitness (e.g. larger number of smaller eggs are likely to increase mortality rate). One could thus say that 'fitness' refers to the biological performance at the level of an organism (to population or species), just as 'adaptation' refers to the performance at the level of an apparatus. A rodent jaw apparatus may thus be adapted for generating larger bite forces, facilitating access to new food resources that provide the energy for an increased and healthy offspring, thus fitness. However, as the organism is a complex and highly integrated architecture of apparatuses, it is the net result of increased or decreased performances of the individual apparatuses that when combined, determine the fitness effect. That implies that even maladaptations for particular functions can still be retained during evolution, as long as it does not undermine fitness (e.g. although the enlarged tail feathers of a male peacock are maladaptive for sustained flight performance, it is outweighed by its benefit for mate attractiveness and is thus selected for through sexual selection) [53].

\subsubsection{Non-adaptive evolution}

Evolutionary changes in phenotypes may also result without actual natural selection, and thus without a selection on functional performance. Several mechanisms have been suggested that can explain this non-adaptive, neutral evolution, where random effects induce these changes (e.g. founder effects, bottle necks, genetic drift). That implies that what seems as optimized biological solutions to particular problems may come with a set of traits that have nothing to do with the optimization process.

Also nested interactions within the genotype-phenotype map may falsely give the impression of traits being optimized through adaptive evolution. Linkages between genetic maps for different traits (called epistatic interactions) imply that when natural selection acts upon one of the traits' performance, the other trait will change with it, independent of whether it comes with a performance improvement or not. Such pleiotropic effects [54] can thus give the impression of traits being optimized, whereas they are only the byproduct of the evolutionary optimization of another trait. This can create a different phenotypic landscape for the byproduct trait (the epigenetic landscape), which may differ from the adaptive landscape resulting from natural selection [55]. Not only at the genetic level, but also at the structural and functional levels, such linked interactions exist. Just as in architecture, many examples of evolutionary spandrels (coined by Gould and Lewontin [46]) have been illustrated, such as bipedal locomotion in lizards [56], the origin of the vocal system in birds [57] or the obstetric characteristics of the human pelvis [58].

\subsection{Constrained evolution}

It should be clear now that it is too ideal to state that 'everything goes in evolution'. Evolution is constrained in so many ways (of which some are illustrated below), that evolutionary 'optimization' is most likely to result in a suboptimal solution. This totally undermines the BID dogma, as if "evolution has resolved many of nature's challenges leading to lasting solutions with maximal performance" [43]. As Speck and Speck [32] stated, "traits that have arisen through evolutionary processes, which depend on myriad factors such as the availability of genetic variation, are rarely perfect from an engineering perspective". Many types of evolutionary constraints are known, acting at many levels, such as gene expression, gene functionality, cell differentiation, tissue interactions, genetic ancestry, mechanical trade-offs, etc. This is all under the control of the so-called 'genotype-phenotype map', which explains why not all parts of biological morphospace have been filled during evolution (e.g. birds lack hairs, mammals don't have feathers) [59-61]. The underlying cause of why not all parts of morphospace have been occupied can be approached in two ways: (1) as a kind of force limiting the directionality of evolution, and (2) as a pattern illustrating that certain evolutionary trajectories are just not available for selection (because of the lack of available genetic variation) [41, 62]. A wrong assumption would be that unoccupied parts in biological morphospace simply reflect suboptimal designs that were outcompeted by the ones that do exist. Actually, both from an engineering and natural evolutionary perspective, virtually exploring non-existing phenotypes and their performance can provide answers to both questions on BID optimization and adaptive evolution [63]. 
As a red line running through evolution, there is the phylogenetic constraint. That simply reflects the genetic line of ancestry, implying that any evolutionary transformation of a population or species has to start from the available genetic and inherited signature. Whereas in BID, engineers can start from any preferred starting point, that is not the case for biological designs. That implies that if the starting point was not optimal for a particular biological solution, any evolutionary trajectory from that point is likely to remain somehow suboptimal. Just as this genetic blueprint sets the starting point for a population to evolve, the genetic machinery of an undifferentiated, single cell (i.e. the zygote or fertilized egg cell) sets the starting point and limits for an embryo to develop. Many types of such developmental constraints are known to restrict developmental plasticity, such as non-viable genetic programming during embryogenesis, canalization of genetic variability into constrained phenotypic variability, to hierarchical linkages between developmental units within an embryo (modularity). One of the intriguing aspects of development is how genetic variation gets translated into phenotypic variation, which is clearly not a one-to-one or consistent process. Cases exist where subtle genetic variation creates substantial morphological variation, such as in domesticated dogs [64] or beaks of Darwin finches [65]. On the other side, substantial genetic variation may result in subtle phenotypic variation (e.g. highly similar African mole-rat species substantially differ in chromosome number [66]). Additionally, genetic canalization during development funnels genetic diversity towards a certain level of constancy in phenotypes, which has been considered as an adaptive buffering response (e.g. to cope with negative effects of some mutations) [51]. Part of the mismatch between genetic and phenotypic variability lies in the types of genes involved, whether they are regulatory genes controlling other genes, or not. Minor changes in regulatory genes can accelerate evolutionary changes in a phenotype and its functioning, but only if they are compatible with the complete cellular machinery that controls development (mutations in regulatory genes are more likely to result in non-viable or arrested embryogenesis) [67, 68]. This regulation also exists between developmental units that comprise a developing embryo, where both new modules and new linkages between existing modules arise as the embryo undergoes tissue and organ differentiation. Similar as 'modularity' in design automation is reflecting an increased independency of units of a complex system (by reducing couplings between units, and hence allowing more evolutionary flexibility) [69], does developmental modularity in biology arise from an increased ratio of intramodule integration compared to inter-module integration [67, 70]. They also converge onto the idea that modularity 'comes at the expense of optimal performance', [69]. Yet, the underlying control mechanisms for this intra- and inter-module integration follows substantially different rules and constraints. Biological modularity is generally mediated through cell signalling (e.g. cells of the apical ectodermal ridge in a developing limb bud signalling with underlying mesodermal cells to control musculoskeletal development, or bone-forming and -degrading cells being influenced by local regimes of mechanical loading) [71, 72]. Paradoxically, such biological modularities can both be constraining (modules control each other) and increasing variability (modules influence each other variably). Additionally, also the level of integration itself has been shown to be variable as well, where intensity in mechanical usage can alter the dependency of modules, even in an adaptive manner [73].

For BID thinking, a more biological interpretation of both canalization and modularity could benefit BID optimization. First, the process of canalization and existence of developmental modularity, where genetic variation is funneled into a limited set of phenotypes, is highly relevant for BID researchers to consider, as it "makes unavailable certain phenotypes that might otherwise be adaptively advantageous" [67]. Second, within-module integration in biology may depend both on the genotype and the phenotype, whereas in engineering, only the phenotypic integration (materials) seems relevant $[74,75]$. As such, variability of a single module relies on a substantially different selection process of where to work within biology vs engineering, as well as the selection process itself differs (evolutionary genotype and developmental constraints in biology versus material properties in engineering). Comparing the rodent lower jaw dentary (being a single bone made of the same biomaterial) with a beam, the beam could be considered as a single module in engineering. Yet, the mouse mandible is comprised of different developmental modules that interact with each other developmentally in a hierarchical and variable manner [76]. Also the integrative hierarchy between modules is important to consider, as some modules may be more integrated with each other than others. Sometimes, evolution is incorrectly considered to act equally at all levels. It could be a challenge for BID researchers to improve design adaptability through modularity, by relying on feedback between modules and from the environment.

Does this mean that only biological systems are constrained, whereas engineering approaches in BID are not, or are totally dissimilar? The underlying mechanism and strategy may differ, but the complexity and intensity of selection may be similar. Just as evolutionary history guides organismal innovations, so does technological history define the 
scale of technological development. Especially relevant are constraints imposed by human technologies (e.g. from limitations in computing power to manufacturing processes). Still, a major difference between evolutionary vs engineering constraints lies in their driver, i.e. genetically imprinted history and random influences versus a wellconsidered choice by engineers.

\section{EVOMIMETIC DESIGN THINKING}

In an attempt to even further stimulate collaborations between engineers and biologists, the plea in this paper is to highlight underexplored potentials towards a proper BID optimization. BID thinking could be upgraded by a more elaborate integration of actual evolutionary processes, and thereby by identifying and thus removing underlying limitations in BID solutions .... hence, BID thinking 2.0 or here coined 'evomimetics'. Not only can the untangling of biological structures and functions open up new insights for BID thinking, also the reverse is true: reverse biomimetics can open up new avenues for studying evolutionary processes and how adaptations have arisen in the natural world [63, 77, 78]. As Meijer, et al. [79] perfectly formulated: "nature can serve as a template for future designs, given that the proper questions are asked and the potential pitfalls are identified. The most important pitfall to consider is the fact that nature does not strive for optimality".

With respect to avoiding phylogenetic constraints in BID optimization, a powerful tool applied by evolutionary morphologists to identify the nature of true adaptations may also be very useful to apply in BID, i.e. the study of convergent evolution. Considering that evolution works in a parsimonious way (i.e. the simplest solution is the most likely one), chances are rather low that similar phenotypes arose across unrelated evolutionary lineages performing similar functions, without the similarities reflecting adaptive solutions. The forelimb in bats, pterosaurs and birds are a perfect example of phylogenetic constraints versus adaptive solutions: they all have a highly similar, wing-shaped musculoskeletal architecture, yet they differ at some levels that reflect their different ancestry (e.g. birds lost multiple fingers, which they inherited from their theropod dinosaur ancestor) [80]. Also by comparing relationships between ecological variation across species and the associated functional-morphological variation, could be very powerful to identify the core traits that emerged in response to particular biological problems [81].

Evomimetics could rely on methods in design automatization and optimization. Computational approaches using virtual designs outside the natural morphospace would allow to test whether nature has indeed reached a true optimum, or whether its suboptimal end product is the result of a variety of constraints. Through optimization modelling, virtual phenotypes that reach optimal performance could be generated and evaluated to what degree they match with natural performance. Identifying the differences could then provide insights in evolutionary constraints in the biological designs. For example, using robotic systems (with both morphology and controller allowed to evolve) would prove to be very instrumental for studying evolutionary constraints in musculoskeletal systems [69]. Experimental work showed that quite distinct solutions emerged from simulations to perform rather simple functions (e.g. bipedal locomotion). It is a fact that the last decade, biological and engineering sciences are converging towards each other substantially through novel computational methods, even when tackling biological-evolutionary questions. The same computational simulation methods commonly used to design and improve prototype performance in engineering, are being used to test specific hypotheses on adaptive evolution, hypotheses otherwise impossible to test by relying on biological organisms only. Even more strikingly, the reason why this is done is exactly the same as for BID thinking: to control for individual traits so that their specific functional contribution in an otherwise complexly coupled combination of traits can be disentangled. In biological research, this is done to test to what degree structural and functional constraints exist, or whether functional performance is non-constrained (i.e. optimal), relying on finite element modelling [82], multi-body dynamics analysis [83, 84], computational fluid dynamics [85] or dispersal behavior simulations [86].

A first step in providing workable tools in evomimetics would be to better standardize correspondence between biological terminologies (with their nuances and evolutionary contexts) and engineering analogies, by elaborating on the existing databases and searching algorithms that facilitate the identification of BID solutions to existing engineering problems [26]. This could be expanding the Thesaurus for Bioinspired Engineering Design or the BioTRIZ with a more elaborate biological context and nuances of the included terminologies [35, 87]. An additional layer of filtering could be added to several of the existing BID problem-to-solution decision trees (biomimicry toolboxes), such as the Concept-Knowledge workflow, BioTriz, AskNature taxonomy, Biologically Inspired 
Adaptive System Design tool, etc., to also incorporate possible evolutionary constraints, in order to arrive at not only the best possible BID solution, but also at identifying potentials for further optimization [25, 31, 88-91].

Does this all imply that evomimetics would only be relevant for a solution-driven approach ('biology push'), rather than the more practical problem-driven one ('technology pull') [23]? Not at all. Being able to more efficiently identify the underlying evolutionary constraints that prevented a biological design to reach optimized functionality towards a particular problem, may even speed up the process of finding the best BID solution for a technical problem. Essentially, three key components are needed once a particular engineering function of interest has been properly identified: (1) a database with known processes that can constrain adaptive evolution; (2) a database on metadata that defines at what levels and in what particular manner these constraining processes can influence natural designs; and (3) proper computational tools that link both datasets to generate a useful tool pack of engineering analogies, but in such a way that contextual information in each of those natural processes is preserved as much as possible [31].

\section{CONCLUSIONS}

To the question on whether we can "outperform natural evolution" [69], the answer is yes. It does require a not so straight-forward task of identifying possible reasons whether or not, and to what degree evolution resulted in a suboptimal BID solution. The basic toolbox exists to do just that, the knowledge too (with biologists and engineers). Tan, et al. [26] emphasized that the challenge in biomimetic engineering is to develop methods that "enable us to analyze the characteristics of different biological species in a biota or even in an ecosystem to support the innovations at the strategic, methodological, and organizational levels." Add the genotype-phenotype map in a historic-evolutionary wrapping, bring the biologists in full interaction with engineers, and evomimetics is born. This provides a rather conceptual framework, that further needs to be integrated with human technologies that outperform these biological limitations, as well as match up with technological constraints.

\section{ACKNOWLEDGEMENTS}

Special thanks to Michael M. Porter for his critical and constructive feedback on the manuscript.

\section{REFERENCES}

[1] Cohen, Y. H., and Reich, Y., [Biomimetic design method for innovation and sustainability], Springer, Switzerland (2016).

[2] Benyus, J. M., [Biomimicry: innovation inspired by nature], Harper Perennial, New York (2002).

[3] AERTEC Solutions, "Airbus Beluga," (2017).

[4] Brier, B., Vinh, P., Schuster, M., Mayforth, H., and Chapin, E. J., "A Radiologic Study of an Ancient Egyptian Mummy with a Prosthetic Toe," Anatomical Record-Advances in Integrative Anatomy and Evolutionary Biology 298(6), 1047-1058 (2015).

[5] Baniqued, P. D. E., Dungao, J. R., Manguerra, M. V., Baldovino, R. G., Abad, A. C., and Bugtai, N. T., "Biomimetics in the design of a robotic exoskeleton for upper limb therapy," 1933(040006 (2018).

[6] Quinn, S., and Gaughran, W., "Bionics-An inspiration for intelligent manufacturing and engineering," Robotics and Computer-Integrated Manufacturing 26(6), 616-621 (2010).

[7] Grunenfelder, L. K., Herrera, S., and Kisailus, D., "Crustacean-Derived Biomimetic Components and Nanostructured Composites," Small 10(16), 3207-3232 (2014).

[8] Prianka, T. R., Subhan, N., Reza, H. M., Hosain, M. K., Rahman, M. A., Lee, H., and Sharker, S. M., "Recent exploration of bio-mimetic nanomaterial for potential biomedical applications," Materials Science and Engineering: C (2018).

[9] Watanabe, S., Tero, A., Takamatsu, A., and Nakagaki, T., "Traffic optimization in railroad networks using an algorithm mimicking an amoeba-like organism, Physarum plasmodium," Biosystems 105(3), 225-232 (2011).

[10] Zhang, X. G., and Mahadevan, S., "A Bio-Inspired Approach to Traffic Network Equilibrium Assignment Problem," Ieee Transactions on Cybernetics 48(4), 1304-1315 (2018).

[11] Kotzé, P., "Biomimicry: Turning to nature to solve modern day problems," The Water Wheel September/Ocotober(28-31 (2018). 
[12] Nkandu, M. I., and Alibaba, H. Z., "Biomimicry as an alternative approach to sustainability," Architecture Research 8(1), 1-11 (2018).

[13] Schroeder, T. B. H., Guha, A., Lamoureux, A., VanRenterghem, G., Sept, D., Shtein, M., Yang, J., and Mayer, M., "An electric-eel-inspired soft power source from stacked hydrogels," Nature 552(7684), 214218 (2017).

[14] Martín-Palma, R. J., and Lakhtakia, A., "Progress on bioinspired, biomimetic, and bioreplication routes to harvest solar energy," Applied Physics Reviews 4(2), (2017).

[15] Gonome, H., Nakamura, M., Okajima, J., and Maruyama, S., "Artificial chameleon skin that controls spectral radiation: Development of Chameleon Cool Coating (C(3))," Sci Rep 8(1), 1196 (2018).

[16] Yang, W., Chen, I. H., Gludovatz, B., Zimmermann, E. A., Ritchie, R. O., and Meyers, M. A., "Natural flexible dermal armor," Adv Mater 25(1), 31-48 (2013).

[17] Kim, J., and Park, K., "The Design Characteristics of Nature-inspired Buildings," Civil Engineering and Architecture 6(2), 88-107 (2018).

[18] Meyers, M. A., Hodge, A. M., and Roeder, R. K., "Biological materials science and engineering: Biological materials, biomaterials, and biomimetics," Jom 60(6), 21-22 (2008).

[19] Duro-Royo, J., Zolotovsky, K., Mogas-Soldevila, L., Varshney, S., Oxman, N., Boyce, M. C., and Ortiz, C., "MetaMesh: A hierarchical computational model for design and fabrication of biomimetic armored surfaces," Computer-Aided Design 60(14-27 (2015).

[20] Dullemeijer, P., [Concepts and approaches in animal morphology], Van Gorcum \& Comp. B.V., Nederland (1974).

[21] Asma, S. T., [Following form and function], Northwestern University Press, Illinois (1996).

[22] Speck, O., Speck, D., Horn, R., Gantner, J., and Sedlbauer, K. P., "Biomimetic bio-inspired biomorph sustainable? An attempt to classify and clarify biology-derived technical developments," Bioinspir Biomim 12(1), 011004 (2017).

[23] ISO 18458, "Biomimetics - Terminology, concepts and methodology," in ISO 2015, pp. 1-32 (2015).

[24] Lenau, T. A., Metze, A.-L., and Hesselberg, T., "Paradigms for biologically inspired design," Proc. SPIE 10593, Bioinspiration, Biomimetics, and Bioreplication VIII 10593(2), 1-20 (2018).

[25] Ohlander, L., Willems, M., Leistra, P., and Damstra, S., "Biomimicry toolbox, a strategic tool for generating sustainable solutions?," p. 80, Blekinge Institute of Technology, Karlskrona, Sweden (2018).

[26] Tan, R., Liu, W., Cao, G., and Shi, Y., "Creative design inspired by biological knowledge: Technologies and methods," Frontiers of Mechanical Engineering (2018).

[27] Darwin, C., [The origin of species by means of natural selection, on the preservation of favoured races in the struggle for life], 6th ed., John Murray, London (1884).

[28] Rugaber, S., Bhati, S., Goswami, V., Spiliopoulou, E., Azad, S., Koushik, S., Kulkarni, R. D., Kumble, M., Sarathy, S., and Goel, A., "Knowledge Extraction and Annotation for Cross-Domain Textual Case-Based Reasoning in Biologically Inspired Design," in Lecture Notes in Computer Science Goel, A., Díaz-Agudo, M. B., and Roth-Berghofer, T., Eds., 24th International Conference on Case-Based Reasoning (ICCBR) 342-355 (2016).

[29] Vincent, J., "TRIZ as a Primary Tool for Biomimetics," in [Research and Practice on the Theory of Inventive Problem Solving (TRIZ): Linking Creativity, Engineering and Innovation] Chechurin, L., Ed., pp. 225-235, Springer International Publishing, Cham (2016).

[30] El Kirat, K., "Biomechanics of Biomimetic and Bioinspired Systems. Innovation inspired by Nature," Fourth International Conference on Advances in Biomedical Engineering (ICABME) 1-3 (2017).

[31] Kruiper, R., Vincent, J., Abraham, E., Soar, R., Konstas, I., Chen-Burger, J., and Desmulliez, M., "Towards a Design Process for Computer-Aided Biomimetics," Biomimetics 3(3), (2018).

[32] Speck, T., and Speck, O., "Process sequences in biomimetic research," in [Design and Nature IV] Brebbia, C. A., Ed., pp. 3-11, WIT Press, Southampton, UK (2008).

[33] Snell-Rood, E., "Bring biologists to biomimetics," Nature 529(277-278 (2016).

[34] Verraes, W., "A Theoretical Reflection on Some Crucial Concepts in Functional-Morphology," Acta Morphologica Neerlando-Scandinavica 27(1-2), 75-81 (1989).

[35] Nagel, J. K. S., "A thesaurus for bioinspired engineering design," in [Biologically Inspired Design: Computational methods and tools] Goel, A. K., McAdams, D. A., and Stone, R. B., Eds., pp. 63-94, Springer-Verlag, London (2014). 
[36] Koch, P., Peplinski, J., Allen, J. K., and Mistree, F., "A Method of Design Using Available Assets Identifying a Feasible System Configuration," Behav Sci 39(3), 229-250 (1994).

[37] Hirtz, J., Stone, R. B., McAdams, D. A., Szykman, S., and Wood, K. L., "A functional basis for engineering design: Reconciling and evolving previous efforts," Research in Engineering Design 13(2), 65-82 (2002).

[38] Van Wassenbergh, S., Heindryckx, S., and Adriaens, D., "Kinematics of chisel-tooth digging by African mole-rats," The Journal of experimental biology 220(Pt 23), 4479-4485 (2017).

[39] Van Daele, P. A., Herrel, A., and Adriaens, D., "Biting performance in teeth-digging African mole-rats (Fukomys, Bathyergidae, Rodentia)," Physiological and biochemical zoology : PBZ 82(1), 40-50 (2009).

[40] Vincent, J. F. V., "The trade-off: a central concept for biomimetics," Bioinspired, Biomimetic and Nanobiomaterials 6(2), 67-76 (2016).

[41] Roff, D. A., and Fairbairn, D. J., "The evolution of trade-offs: where are we?," Journal of Evolutionary Biology 20(2), 433-447 (2007).

[42] Galis, F., "Why Do Almost All Mammals Have Seven Cervical Vertebrae? Developmental Constraints, Hox Genes, and Cancer," Journal of Experimental Zoology 285(19-26 (1999).

[43] Bar-Cohen, Y., [Biomimetics: biologically inspired technologies], CRC Press, Boca Raton, USA (2006).

[44] Sellers, W. I., Margetts, L., Coria, R. A., and Manning, P. L., "March of the Titans: The Locomotor Capabilities of Sauropod Dinosaurs," PloS one 8(10), (2013).

[45] Byrne, G., Dimitrov, D., Monostori, L., Teti, R., van Houten, F., and Wertheim, R., "Biologicalisation: Biological transformation in manufacturing," CIRP Journal of Manufacturing Science and Technology (2018).

[46] Gould, S. J., and Lewontin, R. C., "The spandrels of San Marco and the Panglossian paradigm: a critique of the adaptationist programme," Proceedings of the Royal Society of London B 205(1161), 581-598 (1979).

[47] Futuyma, D. J., [Evolutionary biology], Sinauer Associates, Inc., Sunderland, Massachusetts (1998).

[48] Simmons, R. E., and Scheepers, L., "Winning by a neck: sexual selection in the evolution of Giraffe," The American Naturalist 148(5), 771-786 (1996).

[49] Weeks, P., "Red-billed oxpeckers: vampires or tickbirds?," Behavioral Ecology 11(2), 154-160 (2000).

[50] Dawkins, R., [The selfish gene], Oxford University Press, Oxford, UK (1976).

[51] Pagel, M., [Encyclopedia of evolution], Oxford University Press, New York (2002).

[52] Hendry, A. P., Huber, S. K., De León, L. F., Herrel, A., Podos, J., "Disruptive selection in a bimodal population of Darwin's finches," Proceedings of the Royal Society B (2008).

[53] Askew, G. N., "The elaborate plumage in peacocks is not such a drag," The Journal of experimental biology 217(Pt 18), 3237-3241 (2014).

[54] Hu, H., Uesaka, M., Guo, S., Shimai, K., Lu, T. M., Li, F., Fujimoto, S., Ishikawa, M., Liu, S., Sasagawa, Y., Zhang, G., Kuratani, S., Yu, J. K., Kusakabe, T. G., Khaitovich, P., Irie, N., and Consortium, E., "Constrained vertebrate evolution by pleiotropic genes," Nat Ecol Evol (2017).

[55] Chenn, A., and Walsh, C. A., "Increased neuronal production, enlarged forebrains and cytoarchitectural distortions in beta-catenin overexpressing transgenic mice," Cerebral Cortex 13(6), 599-606 (2003).

[56] Aerts, P., Van Damme, R., D'Août, K., and Van Hooydonck, B., "Bipedalism in lizards: whole-body modelling reveals a possible spandrel," Philosophical Transactions: Biological Sciences 358(1437), 15251533 (2003).

[57] Kingsley, E. P., Eliason, C. M., Riede, T., Li, Z., Hiscock, T. W., Farnsworth, M., Thomson, S. L., Goller, F., Tabin, C. J., and Clarke, J. A., "Identity and novelty in the avian syrinx," Proc Natl Acad Sci U S A (2018).

[58] Mitteroecker, P., and Fischer, B., "Adult pelvic shape change is an evolutionary side effect," Proc Natl Acad Sci U S A 113(26), E3596 (2016).

[59] Wagner, G. P., and Altenberg, L., "Perspective: Complex adaptations and the evolution of evolvability," Evolution 50(3), 967-976 (1996).

[60] Claverie, T., and Wainwright, P. C., "A morphospace for reef fishes: elongation is the dominant axis of body shape evolution," PloS one 9(11), e112732 (2014).

[61] Ward, A. B., and Mehta, R. S., "Differential occupation of axial morphospace," Zoology (Jena) 117(1), 7076 (2014).

[62] Kirkpatrick, M., and Lofsvold, D., "Measuring Selection and Constraint in the Evolution of Growth," Evolution 46(4), 954-971 (1992). 
[63] Porter, M. M., Adriaens, D., Hatton, R. L., Meyers, M. A., and McKittrick, J., "Why the seahorse tail is square," Science 349(6243), (2015).

[64] Boyko, A. R., Quignon, P., Li, L., Schoenebeck, J. J., Degenhardt, J. D., Lohmueller, K. E., Zhao, K., Brisbin, A., Parker, H. G., vonHoldt, B. M., Cargill, M., Auton, A., Reynolds, A., Elkahloun, A. G., Castelhano, M., Mosher, D. S., Sutter, N. B., Johnson, G. S., Novembre, J., Hubisz, M. J., Siepel, A., Wayne, R. K., Bustamante, C. D., and Ostrander, E. A., "A simple genetic architecture underlies morphological variation in dogs," PLoS Biol 8(8), e1000451 (2010).

[65] Abzhanov, A., Protas, M., Grant, B. R., Grant, P. R., and Tabin, C. J., "Bmp4 and morphological variation of beaks in Darwin's finches," Science 305(1162-1464 (2004).

[66] Van Daele, P. A. A. G., Faulkes, E., Verheyen, E., and Adriaens, D., "African mole-rats (Bathyergidae): A complex radiation in tropical soils," in [Subterranean Rodents: News from Underground] Begall, S., Burda, H., and Schleich, C. E., Eds., pp. 357-373, Springer-Verlag, Heidelberg (2007).

[67] Walsh, D. M., "Fit and diversity: Explaining adaptive evolution," Philosophy of Science 70(2), 280-301 (2003).

[68] Holland, P., "Homeobox Genes in Vertebrate Evolution," Bioessays 14(4), 267-273 (1992).

[69] Lipson, H., "Evolutionary robotics and open-ended design automation," in [Biomimetics: biologically inspired technologies] Bar-Cohen, Y., Ed., pp. 129-155, CRC Press, Boca Raton, USA (2006).

[70] Klingenberg, C. P., "Morphological Integration and Developmental Modularity," Annual Review of Ecology Evolution and Systematics 39(115-132 (2008).

[71] Tamura, K., Kuraishi, R., Saito, D., Masaki, H., Ide, H., and Yonei-Tamura, S., "Evolutionary Aspects of Positioning and Identification of Vertebrate Limbs," Journal of anatomy 199(195-204 (2001).

[72] Serrano, J. C., Cora-Cruz, J., Diffoot-Carlo, N., and Sundaram, P. A., "Adaptive responses of murine osteoblasts subjected to coupled mechanical stimuli," J Mech Behav Biomed 77(250-257 (2018).

[73] Anderson, P. S. L., Renaud, S., and Rayfield, E. J., "Adaptive plasticity in the mouse mandible," Bmc Evolutionary Biology 14((2014).

[74] Kimmel, C. B., "Skull developmental modularity: a view from a single bone - or two," J Appl Ichthyol 30(4), 600-607 (2014).

[75] Melo, D., Porto, A., Cheverud, J. M., and Marroig, G., "Modularity: Genes, Development, and Evolution," Annual Review of Ecology, Evolution, and Systematics, Vol 47 47(463-486 (2016).

[76] Klingenberg, C. P., Mebus, K., and Auffray, J.-C., "Developmental integration in a complex morphological structure: how distinct are the modules in the mouse mandible?," Evolution and Development 5:5(522-531 (2003).

[77] Gravish, N., and Lauder, G. V., "Robotics-inspired biology," The Journal of experimental biology 221(Pt 7), (2018).

[78] Porter, M. M., and Ravikumar, N., "3D-printing a "family" of biomimetic models to explain armored grasping in syngnathid fishes," Bioinspir Biomim (2017).

[79] Meijer, K., Moreno, J. C., and Savelberg, H. C. M., "Biological mechanisms as models for mimicking: sarcomere design, arrangement and muscle function," in [Biomimetics: biologically inspired technologies] Bar-Cohen, Y., Ed., pp. 41-56, CRC Press, Boca Raton, USA (2006).

[80] de Bakker, M. A., Fowler, D. A., den Oude, K., Dondorp, E. M., Navas, M. C., Horbanczuk, J. O., Sire, J. Y., Szczerbinska, D., and Richardson, M. K., "Digit loss in archosaur evolution and the interplay between selection and constraints," Nature 500(7463), 445-448 (2013).

[81] Arnold, S. J., "Morphology, Performance and Fitness," American Zoologist 23(2), 347-361 (1983).

[82] Ross, C. F., "Finite element analysis in vertebrate biomechanics," Anat Rec A Discov Mol Cell Evol Biol 283(2), 253-258 (2005).

[83] Praet, T., Adriaens, D., Van Cauter, S., Masschaele, B., De Beule, M., and Verhegghe, B., "Inspiration from nature: dynamic modelling of the musculoskeletal structure of the seahorse tail," International journal for numerical methods in biomedical engineering 28(10), 1028-1042 (2012).

[84] Curtis, N., "Craniofacial biomechanics: an overview of recent multibody modelling studies," Journal of anatomy 218(1), 16-25 (2011).

[85] Van Wassenbergh, S., van Manen, K., Marcroft, T. A., Alfaro, M. E., and Stamhuis, E. J., "Boxfish swimming paradox resolved: forces by the flow of water around the body promote manoeuvrability," J R Soc Interface 12(103), (2015). 
[86] Travis, J. M. J., Mustin, K., Barton, K. A., Benton, T. G., Clobert, J., Delgado, M. M., Dytham, C., Hovestadt, T., Palmer, S. C. F., Van Dyck, H., and Bonte, D., "Modelling dispersal: an eco-evolutionary framework incorporating emigration, movement, settlement behaviour and the multiple costs involved," Methods in Ecology and Evolution 3(4), 628-641 (2012).

[87] Vincent, J. F. V., Bogatyreva, O. A., Bogatyrev, N. R., Bowyer, A., and Pahl, A. K., "Biomimetics: its practice and theory," J R Soc Interface 3(9), 471-482 (2006).

[88] Pidaparti, R. M., and Nagel, J. K. S., "C-K Theory based bio-inspired projects in a sophomore design course," in ASEE Southeastern Section Conference, pp. 1-6, American Society for Engineering Education (2018).

[89] Hatchuel, A., and Weil, B., "A new approach of innovative design: an introduction to C-K theory," International Conference of Engineering Design ICED 1-15 (2003).

[90] Hooker, G., and Smith, E., "AskNature and the biomimicry taxonomy," Insight 19(1), 46-49 (2016).

[91] Arroyo, M., Huisman, N., and Jensen, D. C., "Exploring Natural Strategies for Bio-Inspired Fault Adaptive Systems Design," Journal of Mechanical Design 140(9), (2018).

*dominique.adriaens@ugent.be; phone 329 2645219; fun-morph.ugent.be 